\title{
A Survey of Communication Content in Software Requirements Elicitation involving Customer and Developer
}

\author{
Noraini Che $\mathrm{Pa}^{1}$ and Abdullah Mohd Zain ${ }^{2}$ \\ ${ }^{1}$ Department of Information System, Faculty of Computer Science and Information System, Universiti \\ Putra Malaysia, Malaysia \\ ${ }^{2}$ Programming and Software Technology Research Group, Faculty of Information Science and Technology, \\ Universiti Kebangsaan Malaysia, Malaysia
}

\begin{abstract}
At the heart of software requirements elicitation lies the communication between customer and developer. There are several valuable components of communication such as medium, sender, receiver, and messages, which relates to the input and output from both parties. Most of these messages are delivered through incompletely, inconsistently or inaccurately defined communication medium. This study has been done to look into the communication content of the current communication practices between developer and customer in Malaysia. The results of this study revealed some important notes on the practices of communication content during software requirements elicitation process in Malaysia.
\end{abstract}

Keywords: requirements elicitation; software requirements specification; communication content.

\section{Introduction}

In general, organization is complex, hence identifying the requirements are especially difficult. In addition, software requirements always change from time to time. Requirements elicitation involves the communication process between customer and developer during the analysis phase in software engineering. There are several important components under consideration during communication, such as the medium, sender, receiver, and the content of messages, which relates to the input and output from both parties. Such information by the customer, which is often delivered verbally and not in writing, will be used to produce Software Requirements Specification document (SRS). At present, several studies have been conducted on the practices of requirements elicitation but none has looked into the communication content between customer and developer.

In practice, communication activity involves messages transmission from sender to receiver, whreby the discussion topic revolves around domain application (Drake et al 1993), business requirements, system barrier and others problems (Paetsch et al.2003). While messages are in the form of information and knowledge, knowledge is

Copyright (C) 2011 Noraini Che Pa and Abdullah Mohd Zain. This is an open access article distributed under the Creative Commons Attribution License unported 3.0, which permits unrestricted use, distribution, and reproduction in any medium, provided that original work is properly cited. Contact author: Noraini $\mathrm{Che} \mathrm{Pa}, \mathrm{e}-$ mail: norainip@fsktm.upm.edu.my 
difficult to transmit because it belongs to a person who manages the particular knowledge. According to Stary (2002), knowledge of an organisation covers tasks and processes that are carried out by customers. Such information and knowledge are in turn used to produce the software requirements document, which is traditionally viewed as a document that communicates the requirements of the customer to the developer who is responsible to build the system. The collection of requirements and its representation must be understandable by both customer and developer.

The remainder of this paper is organized as follows. Section two will describe in detail the software requirements elicitation process and the related works. Section three will present the survey results from the requirements elicitation between customer and developer as practiced in Malaysia. Finally, Section four will conclude the findings with some indications for future work.

\section{Literature Review}

\section{Software Requirements Elicitation}

According to Coulin et al (2005), requirements elicitation is the process of searching, revealing, acquiring, and detailing of requirements for computer-based systems. This process is complex as it involves various activities, techniques, approaches, and support tools. More often, these processes are carried out repeatedly (Aurum \& Wohlin 2005). Requirements elicitation is also looked as a negotiation process among stakeholders in order to achieve an agreement on the system to be developed. Sommerville (2001) identifies activities involved during requirements elicitation as discovered, negotiation, and documentation. According to Haywood and Dart (1996), these activities may be implemented using bottom-up or top-down approach, based on specific customer problem. Aurum and
Wohlin (2005) state that in general, the processes are made up by four principle activities, which are communication, set priorities, negotiation and cooperation with the stakeholder.

Various techniques have been used for requirements elicitation such as interviews, document analysis, group work, ethnography, prototyping, questionnaires, scenarios, and viewpoint. These techniques may be divided into two categories: the interaction between an individual and the interaction between groups (Duran et al 2004). Interactions between individuals are divided into two types: local and distributed. Local interaction includes prototype, group meetings, and interviews. Whereas distributed interaction involve interaction of interviews, conferences, and meetings through video. Non-personal interaction consists of observation, document analysis and questionnaires. According to Coulin et al (2005), most of these techniques are adapted from various disciplines such as social science and engineering.

Requirements elicitations techniques may also be classified into traditional, group, formal, semi formal, and natural language. In traditional ways, requirements elicitation process are performed face to face such as through interviews, whether individually or in a group among customer or manager. There have been several difficulties conducting interview session such as:

(i) it is time consuming;

(ii) there may exists conflict between user and manager with regards of perception, assumption, problem defined, and even objective of a system and

(iii) different personalities and behavior (Bahn 1995), as well as background and terminology used during communication between both parties (Liou \& Chen 1993). 
Nonetheless, this technique requires direct interaction between both parties; the interviewer and the respondent, which results in quick information exchange. The quality of the information obtained is closely related to the skills of the interviewer.

Basically, there are three forms of interview, which are unstructured, structured, and semi-structured. Unstructured interviews give the respondent the freedom to express opinions, feelings, position, goals, and beliefs of an issue. This form can be used if the interviewer has little knowledge of the domain. The weakness of unstructured interview is the tendency of both parties to focus discussion on only specific topics. A structured form of interview allows the parties to involve and determine the topic in advance. The results from structured interviews are easily analyzed, the process only takes a considerable short time, and best carried out by a new analyst. However, interviewing techniques actually involve high costs and time consuming to prepare the interviews, performing the interviews and analyzing the results of the interview. In some situations, an interview has to be conducted over time and involve several individuals, with different needs and requirements. Finding by Hickey et al (1999) reveals that this technique is not efficient if the number of respondent involves the public and consists of different groups.

Document analysis technique is conducted by reviewing documents and application of an existing system. This technique is most suitable for the renovation of obsolete systems or by a new analyst. The documents involved include design documents, manual systems, as well as forms and files used in the business processes. However, more often the documents involved contain outdated or incomplete, and inconsistent with the current business requirements (Hoffer et al 2008).

Elicitation techniques that involve public participation or occur at the same time for instance meetings, focus groups, and workshops require a designated working group. Hickey et al (1999) and Drake et al (1993) have categorized meeting techniques that involve time and high cost as it requires the involvement of many parties at one time. Focus group is one of the techniques performed in a group interview. This technique involves participation of the customer representatives and the developer to exchange information through discussions (Sommerville 2007). A facilitator will be appointed to ensure that the discussions are conducted smoothly, hence the technique is less suitable for requirement specifications of complex software systems. Meanwhile, workshop is conducted in collaboration consisting of five stages of development, critique, understanding and support, implementation, and delay (Gottesdiener 2003), whereby all participants play a role in every stage of the workshop conducted. This technique is able to produce high quality requirements within a short time.

Prototyping is another requirements elicitation technique that allows user feedback and considers in-depth information, which is considered the most suitable technique for developing the user interface requirements that have not been identified in full. The prototype responds better to uncertain or changing of requirements (Satzinger et al 2002). Two prototype approaches are incremental and throw away. Incremental prototype is the prototype that is built in a small module from the overall user requirements. Unlike incremental, throwaway prototyping does not preserve the prototype that has been developed. There is never any intention to convert the prototype into a working system (Hoffer et al 2008). This technique is used to encourage user to participate in developing the customer requirements and benefits the discussions with customers because it involves a system that is already in existence.

Meanwhile, elicitation through questionnaire requires a clear focus to ensure the information obtained is appropriate. Questionnaires are used to gather information when the project involves many 
respondents and is to be completed within a short time period. The information obtained is usually lack in depth, less authentic, and less interactive. Normally, this technique is best used to obtain information on attitudes, beliefs, and basic features for a system. Other than questionnaire, observations may be performed by observing how users work out the actual business process without their intervention. This technique involves high costs and requires skill to interpret and understand human actions. Often, users tend to change how they work after finding out that they are being observed In addition, interpretation of the observations made by the analyst is subject to influence and personal bias.

Scenario-based elicitation technique is basically a summarized description of the system as described in the beginning of the process, along the process, and at the end of the process. The scenario is served in the form of a story and contains information on the process, actions and interactions of users with the system. However, this technique does not show the internal structure of a system although it may be used to understand and to validate the requirements.

The most commonly used communication type during requirements elicitation processes are verbal, written, and mediator (Saiedian \& Dale 2002, Coughlan et al 2003). The medium chosen is important to assure the types of messages received are similar to the actual messages that were delivered. Usually, the chosen method is in favors of communication with fast feedback time, clear, no conflict, and easy to understand. Many customers and developers alike use natural language to communicate during requirements elicitation process. However, this method poses some problems such as differences in pronunciation, expression, human emotion, and ambiguous information. (Loucopoulos and Champion 1992).

\section{Communication Content among Software Developers in Malaysia}

The general objective of this survey is to identify communication content that relates to requirements elicitation activities between customer and developer specifically in

Malaysia. The questionnaire encompasses questions on communication content and the appropriate tools used to support the elicitation activities.

The specific objectives of this study are:

(1) to determine the input and output of requirements elicitation process and

(2) to recognize the actual processes involved during requirements elicitation. To achieve the above objectives, the following are some research questions that need to be addressed:

1. What is the source of requirements elicitation for communicating requirements during requirements elicitation in Malaysia?

2. What are the method and support tools used in preparing for software requirements specification document?

3. What are the roles of users' involvement when performing requirements elicitation?

\section{Stakeholder Background}

The methods of data collection in this survey are through postal, e-mail, and interviews. The respondents involved are software developers from various sectors in Malaysia. Questionnaires are appropriate because our data collection involves public respondents where the distribution of the respondents is scattered. The selection of respondents is determined based on their position and experience in requirements elicitation 
activity during system development. Participations came from various agencies that are categorized as government, semigovernment, private agencies with
Multimedia Super Corridor (MSC) status and without. Table 1 shows the background of the respondents who participated in this study.

\section{Table 1: Background Respondent Selection}

\begin{tabular}{ll}
\hline Position & Frequency (\%) \\
& \\
Project Leader & $22(52.4 \%)$ \\
Software engineer & $1(2.4 \%)$ \\
System analyst & $9(21.4 \%)$ \\
Programmer & $2(4.8 \%)$ \\
Others & $8(19.0 \%)$
\end{tabular}

\section{Sector}

Government

Private (MSC status)

Private (Non-MSC status

\section{Experience(years)}

Less than 3 years

3 to 10 years

11 to 20 years

$10(21 \%)$

21(50\%)

$11(26.2 \%)$

\section{Experience(position)}

$\begin{array}{ll}\text { Analyst } & 12(28.6 \%) \\ \text { Designer } & 3(7.1 \%) \\ \text { Developer } & 2(4.8 \%) \\ \text { Analyst and Designer } & 4(9.5 \%) \\ \text { Designer and Developer } & 5(11.9 \%) \\ \text { Analyst, Designer and Developer } & 16(38.1 \%)\end{array}$

\section{Types of Software}

Business Application

Database System

Utility Program

Education Software

Communication Software

Productivity Equipment

Computer Others.

$$
\begin{aligned}
& 21(50 \%) \\
& 10(23.8 \%) \\
& 4.8 \% \\
& 4.8 \% \\
& 1(2.4 \%) \\
& 2(2.4 \%) \\
& 5(11.9 \%)
\end{aligned}
$$

Total

$42(100 \%)$
In the following sections, we will present the analyses performed on the information gathered from 42 responses. The results of the survey were then analyzed using SPSS. 


\section{Results}

Table 2 shows the content and criteria investigated in the survey. There are 5 categories of content, which are the

requirements sources, analysis and modeling, prototype, SRS, and user involvement.

Table 2: Content and Criteria Investigated

\begin{tabular}{|l|l|}
\hline Content & Criteria Investigated \\
\hline Sources & $\begin{array}{l}\text { - Customers do not know how to } \\
\text { specify their needs }\end{array}$ \\
& $\begin{array}{l}\text { - Difficult to understand the customer's } \\
\text { needs }\end{array}$ \\
\hline $\begin{array}{l}\text { Analysis and } \\
\text { Modeling }\end{array}$ & $\begin{array}{l}\text { - } \text { - Methodology used } \\
\text { - Support tools used }\end{array}$ \\
\hline Prototype & $\begin{array}{l}\text { - Type of prototype } \\
\text { - Support tools used }\end{array}$ \\
\hline SRS & $\begin{array}{l}\text { - Content of SRS } \\
\text { - Support tools used }\end{array}$ \\
\hline $\begin{array}{l}\text { User } \\
\text { involvement }\end{array}$ & $\begin{array}{l}\text { - User prefers to validate the interface } \\
\text { rather than the system functionalities }\end{array}$ \\
\hline
\end{tabular}

\section{Requirements Sources}

Requirements sources are information that are gathered from the customers. These refer to customer needs for new implementations or even upgrades. From the analysis, it is found that numerous sources from customers were used in process identification requirements. The survey result shows $69.0 \%$ of respondents chose the work process as their main information source to identify the software requirements. Other sources used are based from existing system (50.0\%), $50.0 \%$ from the organization rules, $50.0 \%$ from expert knowledge, $42.9 \%$ from documents, and $4.8 \%$ from others source (refer to Table 3 ).

Table 3: Sources of Requirements

\begin{tabular}{|l|l|l|}
\hline Sources & Number & Percentage (\%) \\
\hline Work Process & 29 & 69.0 \\
\hline Expert Knowledge & 21 & 50.0 \\
\hline Organization Rules & 21 & 50.0 \\
\hline Existing System & 21 & 50.0 \\
\hline Document & 18 & 42.9 \\
\hline Others & 2 & 4.8 \\
\hline
\end{tabular}

Many organizations choose and modify their requirements sources in accordance with technology changes. Besides, sources of project are also influenced by changes of external factors such as economic, politic, social, regulations, financial, psychology, history, and geography. For example, an organization that practices a bureaucratic 
system often faces difficulty in gathering requirements as compared to other nonbureaucratic organizations. Changes of management and political pattern in an organization also influence in delivering the requirements sources. Such new changes may cause customer to feel unhappy and unable to accept. Nonetheless, changes in requirements and scope will rarely affect the information delivered as delivered through email, telephone or interview.

\section{Analysis and Modeling Requirements}

This process includes refining and modeling the requirements. From the analysis, (see Fig.
1) the results of the study show that respondents prefer to use Structured System Analysis and Design Method (SSADM) as compared to Object Oriented Analysis (OOA) with small percentage of preference on internal methodology. This is probably because the traditional method is easy to understand and represent the actual customer requirements. The survey shows that although $71.4 \%$ of developers do not use any specific software to analyze and model the requirements, $28.6 \%$ of them have considered using the Rational Rose, Enterprise Architect or Microsoft Visio.

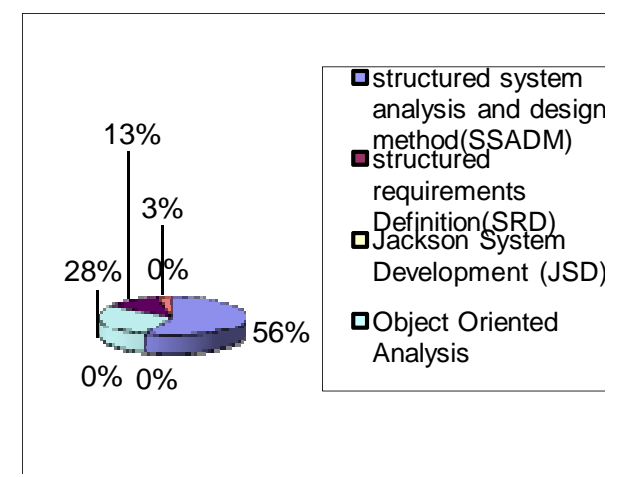

Fig 1. Methodology Used for Software Requirements Analysis and Modeling

\section{Prototype}

Normally in practice, a tool is used to get feedbacks on software requirements as specified by the developer. This type of feedback is used to examine and guarantee the consistency, completeness, reality and accuracy of software requirements.
According to Sommerville (2001), this includes checking the requirements document.

From the analysis, it is shown that $71.4 \%$ of developers used prototype techniques to validate their requirements and $28.6 \%$ chose other techniques (refer Table 4).

Table 4: Techniques of Prototype

\begin{tabular}{|l|l|l|}
\hline Prototype & Number & Percentage (\%) \\
\hline Used & 30 & 71.4 \\
\hline Do not Used & 12 & 28.6 \\
\hline Total & 42 & 100 \\
\hline
\end{tabular}


Feedback from respondents who used prototype is 30 from 42 persons, whereby prototype parts involve user interface, schedule, process flow, and work system. Table 5 shows the use of prototype techniques to communicate system requirement that were developed in effort to seek feedback from customer. Implementation of the prototype involves the programming language and specified software. From the analysis, it is shown that
$86.7 \%$ of developers used programming language to implement the prototype but $13.3 \%$ chose Macromedia Dreamweaver, Microsoft Visio or Microsoft PowerPoint.

Also, most respondents stated that they used combination of requirements part to show the prototype. Study found out as much as $86.7 \%$ presented their prototype for interface, $26.7 \%$ for schedule, $80 \%$ for process flow, and $3.3 \%$ for working system.

Table 5: Parts of Requirements that Demonstrate in a Prototype

\begin{tabular}{|l|l|l|}
\hline $\begin{array}{l}\text { Prototype } \\
\text { Part }\end{array}$ & Number & Percentage (\%) \\
\hline User interface & 26 & 86.7 \\
\hline Table & 8 & 26.7 \\
\hline Process Flow & 24 & 80.0 \\
\hline System Work & 1 & 3.3 \\
\hline
\end{tabular}

\section{Software Requirements Specification (SRS) Documentation}

Because software requirements are often seen as abstract statements of the services provided or the constraints of a system, they are defined in various ways. Software requirements document can also be viewed as a detailed statement that defines the process using formal mathematics of a functional system. According to IEEE (Yang \& Tang 2003), SRS documentation is a term referring to software requirements with:

(i) the capacity required by users to solve a problem or to achieve certain objectives,

(ii) the ability of the system to fulfill the contract, standards, specifications or other and

(iii) a document that reflects the ability to satisfy objective (i) and (ii). Chirinos et al (2004) report that there is actually no consensus on the meaning of software requirements.
Yadav et al (1988) and Whitten et al (2001) present how a requirement is described, which are through:

(i) activities,

(ii) input and output,

(iii) data definition, and

(iv) processing requirements.

In subsequent research, Gregoriades et al (2004) define software requirements as goals to be achieved and consider the implementation through software operating processes, machines, and humans. Software requirements are divided into two types, which are the functional requirements and non-functional requirements. Functional requirements refer to the functions or services provided by the system. This requirement highly depends on the software, potential users, and the type of systems. It is also known as the behavior of the system (Chirinos et al 2004). Meanwhile, nonfunctional requirements refer to the constraints of the system (Paetsch et al 2003). The process of documenting the 
software requirements includes activities such as creating the software requirements specifications (SRS), reviewing the SRS content, and checking the resulting SRS. These activities are carried out to ensure the document that is created adheres to the quality standard and satisfies the customer. Basically, software requirements document is a group of statements that needs to be written by developer (Sommerville 2001). The details of software requirements document depends on the kind of system to be developed and the software development process (Sommerville 2001). There are various standards in existence for requirements document such as the IEEE, ISO 9000, and others. Basic issues in IEEE standard 830-1998 pertaining the SRS document include:

\section{Functionality}

What is the software supposed to do?

\section{External interfaces}

How does the software interact with people, the system's hardware, other hardware, and software?

\section{Performance}

What are the functions of speed, availability, response time and recovery time of various software, etc?

\section{Attributes}

What are the portability, correctness, maintainability, security issues under consideration?

5. Design constraints imposed on an implementation

Are there any required standards in effect, implementation language, policies for database integrity, resource limits, operating environment?

The survey results show that respondents did follow some standard in preparing SRS documentation, among which are from the Institute of Electrical and Electronics Engineers (IEEE), International Standards Organization (ISO) 9000-3, National Standards or internal organization. Analysis of data showed that 53\% respondent follows their own organization standard or at least refer to similar organization in writing the SRS document. While 28\% of respondents do not adopt any formal standard, $13 \%$ of respondents adhered to standard set by IEEE, 3\% adhered to ISO standard 9000-3, while the remaining $3 \%$ adhered to the National Standards.

Further analysis reveals that most of the SRS document content includes the following items:

- Introduction

- Content

- Project background

- System cope and business

- System summary

- Interface

- Output and input

- Process

- Procedure

Meanwhile, only a small number of organizations incorporated the following additional items:

- Change control

- Storage data

- Review

- Validation

As for the tools, software that is used to prepare the SRS document is mainly word processor or specific software. Findings show that $90.5 \%$ respondents used word processor to write SRS and 7.1\% use other specific software, while the remaining 2.4\% use both types of software. Examples of specific software are Microsoft Visio, Microsoft Excel and Microsoft Project. 


\section{User Involvement}

Findings from the survey show that most customers are involved in checking the SRS document. Analysis of data shows that $88.1 \%$ respondent claimed customer involvement in checking on SRS document while 11.9\% claimed otherwise (indicated in Table 6).

Table 6: User Involvement

\begin{tabular}{|l|l|l|}
\hline User & Number & Percentage (\%) \\
\hline Involve not & 37 & 88.1 \\
\hline $\begin{array}{l}\text { Do } \\
\text { involve }\end{array}$ & 5 & 11.9 \\
\hline Total & 42 & 100 \\
\hline
\end{tabular}

Table 7 shows the itemized content of SRS document that are validated by customer. This information is gained after the respondents were requested to list the section of SRS document that requires confirmation by customers. Analysis of data shows that $89.2 \%$ respondents claimed involvement of customer in functional part, $73 \%$ in system scope and business part, $73 \%$ in interface part, $73 \%$ in input and output part, and $73 \%$ other parts. All respondents state that they do not use any specific software to check the SRS document.

Table 7: Parts of SRS that Validate by User

\begin{tabular}{|l|l|l|}
\hline SRS Part & Number & Percentage (\%) \\
\hline System and business scope & 27 & 73.0 \\
\hline Functional & 33 & 89.2 \\
\hline User Interface & 27 & 73.0 \\
\hline Input and Output & 27 & 73.0 \\
\hline Others & 27 & 73.0 \\
\hline
\end{tabular}

\section{Consolidation of the Result}

Based on the survey findings reported in Section 3, the content of communication between the customer and developer during requirements elicitation are investigated in effort to further understand the common practices during the elicitation process. While previous researchers look for technique and sources that is used to generate SRS, there is also researcher that focuses on support tools to facilitate communication between customer and developer during the requirements elicitation process. While previous studies only look into user involvement for requirements validation, this study includes source of communication, user involvement and support tool that are used in performing requirements elicitation.

Overall, the survey conducted is able to provide insights on current communication practices during requirements elicitation activity among software developers in Malaysia. The sources for generating the software requirements were identified by this study. The study also showed that software developers do not use any specific tools to support all activities for requirements during the requirements elicitation process. Survey also shows that there is no specific methodology adopted by the developers to implement the requirements elicitation process. In addition, 
it is found only a handful of developers who use tools to support requirements elicitation.

\section{Conclusion and Future Research}

This paper discusses communication content between the customer and developer during requirements elicitation process in preparing the Software Requirement Specification (SRS) document. The findings show that most developers do not use any support tool in implementing activities during the requirements elicitation process nor do they follow any methodology to perform requirement elicitation. Requirement document is important because it is always taken as the basis for software development, hence a software tool is needed in creating the software requirements document.

One obvious limitation of this study is the use of only one set of questionnaire to be distributed to the developers. In this case, the information gathered is limited to the questions asked. More in-depth information and deeper understanding may be gained if other research methods are used in combination such as focus group and interview. Our future work intend to increase the number of participating companies and to use additional data gathering techniques with the objectives of getting wider and more accurate representation of requirements elicitation practices among industrial practitioners in Malaysia.

There are other interesting issues in communication for requirements to be explored. The issues include medium, personalities, procedures, and communication skill. At the end, our main aim in this endeavor is to facilitate customer and developer to consciously manage future communication during requirements elicitation by looking in-depth of considering the communication content. Effective and clear communication will produce the best software requirement documents, which in turn will produce good software.

\section{References}

Aurum, A. \& Wohlin, C. (2005). "Engineering and Managing Software Requirements," Springler-Verlag Berlin Heidelberg. Germany.

Bahn, D. L. (1995).. "System Designer User Interaction: An Occupational Subcultures Perspective," Proceeding of ACM SIGCPR Conference on Supporting teams, groups, and learning inside and outside the IS function reinventing, 72-80.

Chirinos, L., Losavio, F. \& Matteo, A. (2004). "Identifying Quality-Based Requirements," Information System Management Winter 41(8), 15-26.

Coughlan, J., Lycett, M. \& Macredie, R. D. (2003). "Communication Issues in Requirements Elicitation: A Content Analysis of Stakeholder Experiences," Information and Software Technology 45, 525-537.

Coulin, C., Sahraoui, Abd El Kader \& Zowghi, D. (2005). 'Towards a Collaborative and Combination Approach to Requirements Elicitation within a Systems Engineering Framework,' Proceeding of 18th International Conference on System Engineering, 456-461.

Dawson, L. \& Swatman, P. (1999). "The Use Object-Oriented models in requirements Engineering: A Field Study," Proceeding of. 20th International Conference on Information System, 260-273.

Drake, J. M., Xie, W. W., Tsai, W. T. \& Zualkernan, I. A. (1993). "Approach and Case Study of Requirement Analysis Where End Users Take an Active Role," Proceeding of 15th International Conference on Software Engineering, 177-186.

Duran, A., Benavides, D. \& Bermejo, J. (2004). Applying System Families Concepts to Requirements Engineering Process Definition, Lecture Notes in Computer Sciences 3014, 1-12. 
http://www.lsi.us.es/ dbc/dbc_archivos/pu bs/benavides07-phd.pdf. [10 Julai 2008]. Gottesdiener, E. (2003). "Requirements by Collaboration: Getting It Right the First Time," IEEE Software 20(2), 52-55.

Haywood, E. \& Dart, P. (1996). "Analysis of Sofware Requirements Models," Proceeding of Australian Software Engineering Conference, 131-138.

Hickey, A. M. Dean D. L. \& Nunamaker, J. F. (1999). "Setting a Foundation for Collaborative Scenario Elicitation," Proceedings of the Hawaii International Conference on System Sciences, 26-30.

Hoffer, J. A., George. J. F. \& Valacich, J.S. (2008). Modern Systems Analysis and Design, Fifth Edition. New Jersey, Pearson International Edition.

Lio, Y.I. \& Chen, M. (1993). "Using Group Support Systems and Joint Application Development for Requirements Specification," Journal of Management Information Systems 10(3), 25-41.

Loucopoulos, P. \& Champion, R. E. M. (1992). "Concept Acquisition and Analysis for Requirements Specification," Software Engineering Journal March 1990, 116-123.

Paetsch, F., Eberlein, A. \& Maurer, F. (2003). "Requirements Engineering and Agile Software Development," Proceeding of 12th IEEE International Workshops on Enabling Technologies: Infrastructure for Collaborative Enterprise, 308-313.
Saiedian, H. \& Dale, R. (2000). "Requirements Engineering: Making the Connection between the Software Developer and Customer," Information and Software Technology 42(6), 419-428.

Satzinger, J. W., Jackson R. B. \& Burd S. D. (2002). Systems Analysis and Design. Second Edition, Course Technology Thomson Learning.

Sommerville, I. (2001). Software Engineering. Sixth Edition. Addision Wesley.

Sommerville, I. (2007). Software Engineering. Eighth Edition. Edinburgh,Addision Wesley.

Stary, C. (2002). "Shifting Knowledge from Analysis to Design: Requirements for Contextual User Interface Development," Behaviour \& Information Technology 21(6), 425-440.

Whitten, J. L., Bentley, L. D. \& Dittman, K. C. (2001). System Analysis and Design Methods. 5th Edition. Boston, McGraw-Hill Higher Education.

Yadav, S. B., Bravoco, R. R., Chatfield, A.T. \& Rajkumar, T. M. (1988). "Comparison of Analysis Techniques for Information Requirement Determination," Communications of the ACM 31(9), 10901096.

Yang, H.-L. \& Tang, J.-H. (2003). "A ThreeStage Model of Requirements Elicitation for Web-based Information System," Industrial Management \& Data System 103(6), 398-409. 